\title{
POLÍTICA, ECONOMÍA Y CIENCIA
}

\author{
EN LA CONDICIÓN HUMANA DE ARENDT
}

\author{
POLITICS, ECONOMICS AND SCIENCE
}

IN ARENDT'S THE HUMAN CONDITION

\author{
SANTIAgo Ojeda COUCHOUD* \\ UNED
}

\begin{abstract}
Resumen: En su libro, La condición humana, Hannah Arendt disecciona las presiones a las que la actual sociedad científico-tecnológica somete a la forma tradicional de estar en el mundo de nuestra especie. El núcleo de la obra denuncia cómo la forma más específicamente humana de la vida activa, la acción, se está viendo desplazada por el trabajo e incluso por la labor. Una de las manifestaciones de este desplazamiento es la creciente infiltración de la economía (cuya condición epistemológica como ciencia era indiscutible mientras Arendt escribía) en el discurso político, derivándose de esta infiltración una colonización del espacio público por modos de actuar propios del espacio privado (donde tienen precedencia el trabajo y la labor) que ocasionan su debilitamiento, y con él el vaciamiento de lo que más nos hace humanos. El presente trabajo apunta elementos de crítica a esta concepción de la política, mediante el cuestionamiento del carácter científico de la economía, que puede por ello ser objeto legítimo de la discusión política ocupada en la adecuada configuración colectiva del espacio público.
\end{abstract}

Palabras clave: Condición Humana, Filosofía Política, Labor, Trabajo, Acción, Economía.

*Santiago@ojeda-couchoud.es 
Авsтract: In her book The Human Condition Hannah Arendt dissects the pressures that current scientific and technological society imposes on our traditional way of being in the world. The core of the work decries how the most specifically human form of the active life, action, is being displaced by work, and even by labor. One of the manifestations of this displacement is the growing infiltration of economics (whose epistemic character as a science was undisputed at the time of Arendt's writing) in political discourse, causing a colonization of the public space by ways of behavior more fit for the private space (the traditional space for work and labor), causing its weakening and the erasure of what makes us specifically human. The present work points towards elements for the critique of this conception of politics through the questioning of the scientific character of economics, which would make it a legitimate object of political discussion engaged in the proper collective configuration of the public space.

KEYwORDs: Human condition, political philosophy, labor, work, action, economics

\section{Introducción}

Un libro que se abre con la imagen del primer satélite artificial circunvalando la tierra y se cierra con la admonición de evitar un futuro equiparable en su desesperanza a los peores escenarios postapocalípticos de la serie Mad Max (un futuro en el que habríamos dejado de ser humanos tal como hoy entendemos el término) sólo puede ser un gran libro. Y efectivamente, La condición humana, publicado en 1958, es una obra capital para entender en qué consiste dicha condición, y los peligros que acechan a su continuidad en la época de su publicación.

La utilidad de las grandes obras del pensamiento se encuentra tanto en los caminos que abren y las preguntas que plantean sin llegar a responder como en las leyes que nos descubren y los problemas que resuelven ${ }^{1}$. Se dice que los errores o inexactitudes de una gran creación (si es que puede hablarse de errores en las ciencias del espíritu, cuyo criterio de verdad, como veremos más adelante, estriba menos en su posible correspondencia con la realidad que en su coherencia

\footnotetext{
1 Por supuesto, algunos autores son más propensos a la duda y la pregunta (desde el ilustre iniciador de la tradición filosófica occidental moderna, Sócrates, a los "filósofos de la sospecha" del siglo pasado), mostrándonos en sus obras antes su asombro ante un mundo que se resiste a que le dotemos de sentido que sus intentos de dotarlo ellos mismos, en los que siempre identifican cierta "mala fe". Por la amplitud de los temas que abarca y su alergia a todo dogmatismo Arendt nos parece más próxima a estos autores para los que la actividad filosófica se realiza siempre, usando un término de Javier Muguerza, "desde la perplejidad".
} 
tanto interna como con la red de significados del sistema de signos en que se enuncian) son más fructíferos que las certitudes triviales de las obras menores, y la obra que nos ocupa no es una excepción. El argumento del presente trabajo es que la concepción de la política y la economía que en ella se expresan presenta una inconsistencia, pero el análisis de su inconsistencia, y las perspectivas que nos abre sobre lo que entendemos debe ser el contenido de dichas disciplinas iluminan aspectos de las mismas de gran ayuda para guiarnos en el laberinto de la sociedad postindustrial en que vivimos.

Repasemos pues el marco conceptual que nos propone Arendt para identificar con mayor claridad los elementos que vamos a someter a crítica. En el libro que nos ocupa analiza los diferentes elementos de la "vida activa" 2 , que denomina labor, trabajo y acción. La labor está formada por el conjunto de actividades tendentes a dar continuidad a nuestra existencia física, bien como individuos (comer, dormir) bien como especie (reproducirse, cuidar de la propia descendencia), y es por tanto algo que compartimos con el resto de animales. El carácter animal de esta tarea se refleja en la forma en que tradicionalmente se denomina a quien está involucrado en ella: animal laborans, no todavía alguien específicamente humano. En cuanto al trabajo, está formado por aquellas actividades tendentes a producir un mundo artificial, separado de la naturaleza, del que los humanos necesitamos rodearnos para sobrevivir y prosperar. Sus frutos (el ejemplo paradigmático que da Arendt es una silla o una mesa) son diseñados y construidos con la finalidad de perdurar, en contraste con los frutos del mundo natural, que son perecederos (en la naturaleza sólo las especies perduran, no existen estrictamente individuos). Nos encontramos ya en el ámbito del homo

\footnotetext{
2 La acotación en sí resulta relevante. El otro componente de lo humano es para Arendt la "vida contemplativa", cuyos elementos serían el pensamiento, la volición y el juicio, de los que se comenzó a ocupar en el libro The life of the mind - el término inglés mind, como su equivalente en alemán geist, es notoriamente traicionero para traducir, pudiéndose entender tanto por "mente" como por "espíritu"-, publicado póstumamente. No podemos evitar preguntarnos por qué a la hora de describir la condición humana Arendt considera suficiente limitarse a tratar de la vida activa. ¿consideraría acaso que la vida del espíritu no se ve amenazada por los desarrollos de la moderna sociedad científico-tecnológica? Es difícil de creer, siendo la influencia de la sociedad moderna en fenómenos tan espirituales como la creación artística parte del debate de la época. Por ejemplo, Walter Benjamin publicó su famoso ensayo sobre "la obra de arte en la edad de la reproductibilidad mecánica" ya en 1935, prefigurado en su conferencia "El artista como productor" de 1934. Arendt era una buena conocedora de la obra de Benjamin, hasta el punto de haber editado la traducción al inglés de una colección de sus textos en 1968 bajo el título de "Iluminaciones", que incluye el primero de los ensayos mencionado, y dedicó uno de sus ensayos, "The crisis in culture: its social and its political significance” a analizar el mismo fenómeno, en Arendt (2006).
} 
faber, distinto del animal por su capacidad para modificar de forma permanente el mundo que le rodea, para crearse un mundo más acorde con sus necesidades, pero que no agota ni mucho menos las capacidades humanas. Porque para Arendt el elemento específicamente humano de la vida activa está formado por el conjunto de actividades que agrupa como acción (ya que se puede contratar -o esclavizar- a otros para que se encarguen de la labor, y se puede habitar un mundo artificial al que no se ha contribuido con ningún objeto, pero no se puede vivir sin mostrarse y compartir hechos y palabras con otros humanos). Esa acción es producto en primer lugar de la pluralidad humana, del hecho de que somos todos diferentes individuos a pesar de compartir características comunes. Además, si tanto la labor como el trabajo vienen marcados por estar sujetos al ámbito de la necesidad, sólo en la acción el hombre puede manifestar su libertad, entendida como espontaneidad, hasta el punto de que para Arendt una de sus características es el que sus resultados son necesariamente imprevisibles. Es también por ser un producto de la libertad humana por lo que el sentido de la acción sólo puede determinarse a posteriori, una vez se ha finalizado (como el sentido de una historia sólo se desvela cuando se ha terminado de contar, ya que otra característica de la acción es que constituye el aspecto de nuestra vida "vista desde fuera" digno de ser narrado ${ }^{3}$ ), y por lo que estrictamente carece de finalidad (como mucho, surge de un "principio" general que en ningún caso permite determinarla o dirigirla a priori). Es por ello que de la ejecución de las mismas se derivan dos características igualmente exclusivas de nuestra especie, como son la promesa y el perdón.

Además de proporcionar una tipología de nuestras actividades, Arendt las caracteriza por el ámbito en que éstas se desarrollan. La labor es propia del espacio privado, del hogar. También lo era el trabajo originariamente (cuando las actividades productivas se llevaban a cabo por artesanos que controlaban todo

3 Es relevante que la narrativa tradicional ha dedicado su atención casi exclusivamente a los "hechos y las palabras" de sus protagonistas fuera de su entorno profesional, de modo que en la mayoría de las novelas desde el S XVII a nuestros días la ocupación remunerada de los protagonistas (de haberla, la novela decimonónica se centra en nobles y rentistas que precisamente no necesitan trabajar para vivir, y hemos de esperar a Dickens para disponer de un análisis de las profesiones de la época) permanece en segundo plano, cuando no se ignora por completo. Una prueba de la validez del análisis Arendtiano es el punto hasta el que nuestra época reciente se ocupa precisamente del trabajo de sus personajes y ensalza como héroes (individuos dignos de protagonizar historias) a los directivos de grandes empresas en cuanto tales. Véanse como ejemplo tanto la biografía de Steve Jobs de Walter Isaacson de 2011, como la película dirigida por Aaron Sorkin sobre el mismo sujeto de 2015. Dichas obras habrían sido profundamente paradójicas para la propia Arendt. 
el proceso, antes de que la revolución industrial obligase a juntar innumerables trabajadores en un tipo de lugar común hasta entonces inexistente: la fábrica $\left.{ }^{4}\right)$. Sin embargo, el ámbito propio y diferenciado de la acción es el espacio público, donde hombres libres e iguales se encuentran (y el reconocimiento de dicha igualdad es una de las condiciones de posibilidad de la acción, no hay como llevarla a cabo, por ejemplo, en la corte de un déspota oriental donde sólo uno goza de libertad a costa de la de todos los demás) para intercambiar "grandes hechos y grandes palabras".

El centro del libro se dedica, como avanzábamos, a denunciar el desorden que la modernidad ha traído a este esquema (que se entiende, por ello, como el más adecuado y deseable). En primer lugar, por la pérdida de relevancia de la acción, derivada de la dificultad de encontrarse como iguales en sociedades o abiertamente totalitarias, o subrepticiamente plutocráticas (en que unos pocos controlan todas las decisiones, manteniendo a la mayoría excluida del proceso decisorio por su baja educación o por su embrutecimiento a través de los nuevos medios masivos de formación de conciencias). Derivada de esta dificultad (y en refuerzo de la misma) observamos el creciente debilitamiento del espacio público, facilitado por un lado por la retirada de la mayoría de los ciudadanos a su privacidad (una privacidad cada vez más llena de posibilidades, como veremos) y por otro por la creciente "socialización" del mismo, término con el que Arendt describe la trasposición de valores que históricamente eran propios de la organización familiar al conjunto de la sociedad, como puedan ser la discusión de la mejor forma de asignar los recursos ( originalmente restringida a los recursos del $\operatorname{hogar}^{5}$ ) o la definición de lo que es propio de la persona en función de la clase a que pertenece, negando la posibilidad de expresar su individualidad y unicidad característica del espacio público.

\footnotetext{
${ }^{4}$ El análisis Arendtiano presenta aquí una primera incompletitud, ya que en la historia de la humanidad no faltaban ejemplos de instalaciones pre-industriales en las que grandes cantidades de trabajadores tenían que juntar su esfuerzo, desde la construcción de la grandes obras de ingeniería civil de las primeras civilizaciones, como las pirámides de Egipto o los zigurates de Babilonia, hasta las fábricas medievales de tapices o vidrio, en que empieza a experimentarse con una rudimentaria especialización del trabajo, pasando por los grandes complejos mineros y canteras a cielo abierto de los romanos. Al menos los líderes de dichos trabajadores encontraban la ocasión de realizar grandes hechos (para los que habían reclutado numerosos congéneres) en ausencia de igualdad o de reconocimiento de su humanidad.

5 Pues ése es el origen de la palabra "economía", de oikos (hogar) y nomos (normas, costumbres), o sea, disciplina que trata de determinar las normas para mejor conducir los asuntos del hogar.
} 
Llegamos así al núcleo del argumento que queremos revisar. Las sociedades contemporáneas de la autora (a partir de las cuales se han desarrollado las de nuestros días) nos están volviendo menos humanos al eliminar el espacio para la acción, que es nuestra característica más noble y propia, y la única en que se manifiesta nuestra libertad (entendida, entre otras cosas, como capacidad para generar nuevos inicios, de generar nuevas series de eventos). Esta eliminación es el resultado de permitir la "colonización" (por tomar prestada la terminología propia de la teoría crítica francfortiana en algunos de cuyos satélites hemos visto en la nota 2 que Arendt estuvo interesada) de este espacio por intereses que no le son propios, sean los específicos de la economía (cuya trasposición supone tratar la totalidad de la sociedad como una gran familia, característico de sociedades paternalísticamente autoritarias), sean otros intereses igualmente específicos del ámbito privado que son amplificados a través de los medios de comunicación de masas a través de los cuales más y más de nuestro tiempo consciente se dedica a interesarnos por la vida de personajes enteramente banales (banales por cuanto su interés se limita al hecho de pertenecer a la especie humana en la que se ha eliminado todo elemento de originalidad o individualidad, sin ninguna aspiración por ello a la durabilidad, a la inmortalidad, que para Arendt es definitoria de la acción), sean ficticios o no. Se trata este último de un movimiento cuyo origen sitúa la autora en el "descubrimiento del valor de la intimidad" por Rousseau que desencadenaría el movimiento romántico, pero que el alcance universal de nuestra "cultura del espectáculo" amplifica hasta límites difíciles de prever entonces.

Pero esta crítica a la forma en que están evolucionando las sociedades se apoya al menos en dos presupuestos que hoy nos resultan cuando menos cuestionables, y a su cuestionamiento en más detalle dedicaremos el cuerpo del presente trabajo:

En primer lugar, este esquema da por supuesto un determinado concepto de la economía como ciencia "dura" (antes una Naturwissenschaft que una Geisteswissenschaft en terminología Diltheyana ${ }^{6}$ ). Las ciencias así entendidas son las que permiten realizar predicciones verificables, mediante la aplicación de leyes generales a cada caso particular. El conocimiento de estas leyes generales permite desarrollar aplicaciones prácticas (tecnología) que aumentan nuestro poder sobre la naturaleza y se acaban situando en un

${ }^{6}$ Ver Dilthey (1988) para una caracterización de los diferentes tipos de disciplina, los diferentes criterios de validez que cabe aplicar a los enunciados que las componen y la certeza que cabe asignar a sus predicciones. 
espacio axiológicamente neutro en que la razón que se aplica es puramente instrumental (determinación de los mejores medios para alcanzar fines que la propia razón no puede ni plantearse ni criticar). Bajo este concepto (compartido por la inmensa mayoría de los practicantes de la disciplina económica) es lógico que Arendt excluyese la economía del ámbito de actividades más elevadas a que puede dedicarse el hombre, y que intuyese su capacidad para degradar el nivel del discurso, ya que sobre lo que necesariamente sólo puede ser de una manera no tiene sentido discutir. Es significativa su crítica de la concepción Marxista a lo largo de todo el libro, en lo referente a su falta de originalidad a la hora de suponer un inexistente objetivo común a toda la sociedad (pág. 45, se sirve de la expresión de Gunnar Myrdal de "ilusión comunística" ya presente en los economistas liberales clásicos) y en su confusión entre labor como aquello de lo que el hombre debe librarse (a pesar de ser la fuente última de todo el valor económico) y trabajo como condición de posibilidad de nuestra dignidad (págs. 104 - 105); para ella la explicación en términos puramente económicos nunca podía agotar la caracterización de nuestras acciones, a riesgo de excluir toda posibilidad de considerarnos agentes libres ${ }^{7}$. Sin embargo, defenderemos que la condición epistemológica de la disciplina económica es muy diferente, y que excluye tanto la posibilidad de realizar predicciones fiables como de utilizarse para manipular los asuntos humanos.

En segundo lugar, la visión de Arendt de lo que constituye el ámbito legítimo de la política (apuntado en su seminal Los orígenes del totalitarismo y desarrollado en mayor detalle en libros posteriores como Entre el pasado y el futuro, ¿Qué es la politica?, De la revolución y La promesa de la política) está, como no podía ser de otra manera, influida por la sombra de los grandes regímenes totalitarios a los que había estado expuesta en su juventud (la Alemania nacionalsocialista) y que en el momento en que desarrollaba el núcleo de su actividad intelectual (en los Estados Unidos de América) todavía constituían una amenaza no sólo para la sociedad que la había acogido,

\footnotetext{
7 En Arendt (1998), página 42 podemos leer: “[la economía] puede alcanzar un carácter de ciencia sólo cuando los hombres se hubieran vuelto seres sociales y unánimemente siguieran ciertos patrones de comportamiento", es decir, sólo cuando el ámbito de lo "social" hubiese hecho desaparecer enteramente el espacio público, y eliminado por ello la libertad humana, tendría sentido la economía como ciencia. Sin embargo, la autora no saca de ello la conclusión evidente de que mientras haya espacio público (y el hombre pueda todavía manifestar su libertad) la economía no tendrá en ningún caso el carácter de ciencia, y será por ello un objeto legítimo de debate (como la ética o la crítica literaria).
} 
sino para el conjunto de la especie humana a través de la amenaza de una guerra nuclear total (amenaza esgrimida por la Unión Soviética y sus países satélite). En semejante escenario, Arendt comprendió (y defendió) la política como la disciplina orientada a garantizar espacios de libertad en que los individuos pudieran actuar (en el sentido de acción -handeln-antes explicado), excluyendo de la misma tanto la posibilidad de violencia y coacción como las preocupaciones con el mundo artificial del que debemos rodearnos para sobrevivir (las preocupaciones propias de la economía que mencionábamos en el punto anterior). El motivo es que bajo su comprensión del carácter de la economía, la discusión sobre qué producir y cómo (qué recursos sociales destinar a cada rama de la industria, cuánto invertir y cuánto ahorrar, y sobre todo cómo distribuir el producto igualmente social producido) es una discusión de carácter técnico, en la que se persigue alcanzar verdades "de razón" (matemáticas o científicas, no sujetas por ello a diferencias de opinión) en lugar de verdades "de hecho" (interpretación de acciones, en las que por ello es legítimo mantener diferencias racionales sin que ésas se expliquen por la falta de información o la "mala fe" ${ }^{8}$ de los hablantes), y la discusión y la práctica políticas están reservadas para la persecución conjunta de las verdades factuales. Sin embargo, si tenemos razón en el punto previo y la economía no es, al cabo, más que un subconjunto de la filosofía práctica, cuyas conclusiones se deberían alcanzar precisamente mediante la discusión y el acuerdo, antes a través de la persuasión que de la intuición, la conversación sobre cómo mejor asignar los recursos escasos pertenecería legítimamente al ámbito de la política y sería un asunto cuya consideración enriquece (lejos de empobrecer) la actividad a desarrollar en el espacio público.

\section{De qué hablamos cuando hablamos de economía}

Es fácil criticar la incapacidad de la economía para realizar predicciones fiables (lo que debería ser la piedra angular sobre la que se determina el carácter científico de una disciplina) en la segunda década del siglo XXI, tras el mayor colapso en la producción de los países avanzados en casi cien ańos, colapso que prácticamente nadie vio venir (y que irónicamente tuvo lugar cuando se volvía a hablar del fin de los ciclos económicos tradicionales gracias a nuestra más perfecta

8 Para la diferencia entre verdades de razón y de hecho, véase el ensayo "Verdad y Política" en Arendt (2006), págs. 225-226. 
comprensión de los mecanismos que subyacen a los mercados de bienes, trabajo y capital).

Puesto que no se puede decir que se hayan dedicado pocos recursos de investigación en la disciplina económica (medidos en tiempo de académicos brillantes o en los abultados salarios de analistas y estrategas en las empresas privadas) el que el registro hasta la fecha sea tan pobre ya es una poderosa indicación de que el objeto de estudio ni está suficientemente comprendido (a las pruebas nos remitimos) ni puede llegar a estarlo. La economía no es precisamente una ciencia joven, habiendo formulado sus primeros principios en una forma que podemos llamar moderna los economistas clásicos (Adam Smith, Thomas Malthus y David Ricardo) ya en el S XVIII, sin olvidar que problemas que hoy consideramos completamente económicos, como el del mecanismo de determinación de los precios de cada bien (justo precio), la masa total de dinero en circulación, la tasa de cambio y la cantidad y tipo de comercio deseables entre naciones habían ocupado ya a los escolásticos medievales, a los mercantilistas y a los fisiócratas antes de eso. Tras las contribuciones clásicas, la obra que juega en economía un papel similar al de la teoría de la relatividad general de Einstein en física (y que debería marcar por ello la culminación de una disciplina madura) es la Teoría general del empleo, el interés y el dinero de John Maynard Keynes, publicada en 1936, y que desde entonces ya ha tenido ocasión de ser aceptada universalmente, verificada experimentalmente (al aplicarse para finalmente salir de la que hasta hoy se conocía como "la gran depresión" de los años 30), repudiada (en primer lugar por la escuela austriaca, seguidos poco después por los monetaristas de la escuela de Chicago encabezados por Milton Friedman), "falsada" en el sentido estrictamente científico (con el incumplimiento de sus postulados en las sucesivas crisis del petróleo de los años 70 del pasado siglo) y finalmente reivindicada de nuevo. La última vez que comprobamos, la teoría de relatividad seguía siendo tan universalmente aceptada como el día tras el eclipse de 1919 cuando se comprobó que el sol alteraba la trayectoria de los rayos de luz de la forma predicha?

\footnotetext{
9 No es este el lugar para discutir el carácter igualmente paradigmático de los postulados de la física teórica o el resto de las ciencias "duras", como se viene exponiendo en Kühn (1996) y epígonos. En su interpretación débil (cada avance de la ciencia se encuadra en un conjunto de teorías contemporáneas que limitan los problemas que tiene sentido plantearse y lo que se considera una solución válida a los mismos) es trivial, y en su interpretación fuerte (los diferentes paradigmas que se suceden en el tiempo son inconmensurables entre sí en lo relativo a su capacidad de describir de forma más completa la realidad, ya que al "borrar" las contribuciones de los paradigmas que sustituyen "modifican" el contenido de dicha realidad) es lógicamente inconsistente, y por ello falaz.
} 
Algo hay pues sustancialmente diferente en la naturaleza de lo que tratamos de conocer con las dos construcciones teóricas (la física y la economía), que explique la diferencia en sus resultados.

Utilizando el esquema Arendtiano (que veremos reaprovecha uno más antiguo), podemos distinguir dos tipos de enunciado. El primero, ejemplificado por la autora (tomado de Hobbes) en "los ángulos interiores de los lados de un triángulo suman dos ángulos rectos” sería el enunciado de razón. El segundo, para el que nos ofrece el ejemplo "Trotski jugó un papel destacado en la revolución rusa”, sería el enunciado de hecho ${ }^{10}$. A partir de esta clasificación, podemos preguntarnos de qué tipo es un enunciado de la física como "la masa en reposo del bosón de Higgs es de $100 \mathrm{GeV}$ ”. A primera vista, se trata de un enunciado de hecho: las cosas son así y la única forma de saber si el enunciado es verdadero o no ${ }^{11}$ es "interrogar a la naturaleza" mediante u experimento controlado que nos ayude a determinar la masa de la partícula en cuestión. Sin embargo, dicha interrogación requiere de todo un aparato interpretativo (definiciones axiomáticas de masa, energía, paridad, spin, carga eléctrica y toda una serie de complejas ecuaciones matemáticas que las relacionen) que son tan objeto del experimento como la masa finalmente medida. Puesto que dentro de dicho aparato la masa prevista se obtiene apodícticamente (es un resultado de la teoría cuyo conjunto se termina por confirmar o falsear), el enunciado resulta ser de razón, y no de hecho. Como no podía ser de otra manera, ya que los enunciados de este tipo tienen validez universal, se cumplen sea cual sea el estado del mundo, y por ello permiten extraer consecuencias ciertas tanto de lo que ocurrió en el pasado como de lo que ocurrirá en el futuro. Los enunciados de hecho, por el contrario, sólo permiten describir lo que ocurrió en el pasado, y en general los intentos de

10 La clasificación parece coincidente con la establecida por Kant entre enunciados analíticos y sintéticos, y de hecho Kant es una de las grandes influencias de Arendt. Sin embargo antes que en la Kritik der reinen Vernunft hay que buscar el origen de la clasificación utilizada en la Kritik der Urteilskraft y en el opúsculo "Was heisst: sich im Denken orienteren?", veremos que la diferencia atañe sobre todo a la consideración de los enunciados de las ciencias naturales, que para Kant serían sintéticos a posteriori mientras que como estamos entendiendo el esquema de Arendt serían "de razón” junto a otros juicios entendidos como analíticos a priori por Kant

11 Seguramente no lo es, los resultados publicados en julio del 2014 que confirmaban la detección de la elusiva partícula en el Large Hadron Collider del CERN de Ginebra le asignan una masa entre 125 y $127 \mathrm{GeV}$ (y la importancia de dicho resultado no es que confirmase una predicción individual, sino que, en palabras de los periodistas científicos que cubrieron la noticia, "que confirmaba el imponente edificio teórico del modelo estándar de la física"). 
extraer de ellos consecuencias futuras nos llevan a descuidar una mayor o menor cantidad de cualificaciones y circunstancias adyacentes que vician la predicción.

Pues bien, ante los enunciados típicos de la ciencia económica como "la bajada de los tipos de interés provoca un aumento de la actividad económica y una disminución de la tasa de desempleo" o "la reducción del tipo impositivo entre los que más ingresan conlleva una aumento de la actividad inversora, una mejora del empleo y por ello, un incremento de la recaudación total"12 la mayoría de la gente piensa que se trata de enunciados de razón, similares en todo a los de la física o las matemáticas, y que por ello juzgar su veracidad es una cuestión de obtener la suficiente información que permita probarlos o refutarlos, y determinar así sobre una base firme su validez universal. Se trata sin embargo de enunciados del tipo “Trotski jugó un papel destacado en la revolución”, frágiles por seleccionar un conjunto de eventos de una época concreta en los que se vieron involucrados unas personas libres, enteramente contingentes y afectado del "melancólico carácter accidental" (trostlose Ungefähr) en inolvidables palabras de Kant utilizadas por Arendt ${ }^{13}$.

Defender en economía la existencia misma de supuestas reglas universales ajenas por ello a esa contingencia suele esconder una agenda (en el sentido Marxista) ideológica. Por ejemplo, decir que hay una tasa impositiva que maximiza el crecimiento global de la economía es profundamente falaz, lo que existe es una tasa que maximiza el crecimiento supuestas ciertas condiciones de contorno, y la decisión de si el mantenimiento de tales condiciones es deseable o no requiere de una elección previa para la que el sistema no puede proporcionar directrices. Si algo nos han enseñado las dos últimas décadas es que la premisa implícita (o muy explícita cuando fue necesario defender las políticas derivadas de ella) de los economistas neoliberales partidarios de la continua disminución del estado apoyándose en que "la marea levanta todos los barcos" y el crecimiento era bueno para toda la sociedad por igual (la ahora más infame que famosa trickle down economics) era una falacia: todo el crecimiento de esas dos décadas se ha traducido en incrementos fabulosos en la renta disponible, el consumo y el ahorro del 10\% de la población con mayores ingresos, mientras que las condiciones de vida del

12 De nuevo los dos enunciados presentados son muy probablemente falsos (a la luz de las experiencias históricas recientes, ya que el primero de ellos requiere para no cumplirse de que los tipos estén suficientemente próximos a cero, uns situación que se ha producido con la suficiente poca frecuencia como para no admitir de conclusiones fáciles).

13 En el ensayo “The Concept of History”, pág. 85, Arendt (2006). 
90\% restante siguen siendo prácticamente las mismas que en la década de los 80 (eso sí, ahora la mayoría de ese $90 \%$ tienen iPads, "smart phones", y pantallas de televisión de plasma ${ }^{14}$ ). Al final todos los óptimos que la "ciencia" económica permite perseguir resultan ser óptimos de Pareto ${ }^{15}$, y se puede argumentar que el concepto mismo de óptimo de Pareto, como intento de sustituir valoraciones subjetivas por una regla universalmente aplicable, es profundamente regresivo (el precio de la sustitución es aceptar de forma implícita una regla "ciega" a las consecuencias de la distribución de partida que se pretenda maximizar). La economía no puede ser una ciencia natural por el mismo motivo que la ética no puede ser una ciencia natural. Si se intentan eliminar los valores de la discusión económica nos quedamos sin nada que discutir (y a menudo los intentos de hurtar los valores subyacentes del espacio de lo legítimamente discutible no es ni mucho menos un descuido bienintencionado), y los valores, como toda la tradición de la filosofía analítica no se ha cansado de recordarnos, no son parte del mundo, no admiten ser medidos, pesados o predichos. Los ponemos nosotros en el mundo, pero aquello que elegimos poner es necesariamente contingente.

${ }^{14}$ La referencia es sólo a medias irónica. Cuando la evidencia estadística del fracaso de la trickle down economy se hizo incontestable sus partidarios - personificados por los think tanks ideológicamente próximos al partido republicano norteamericano- contraargumentaron que la estadística no captura adecuadamente la mejora en el nivel de vida de la población en general derivada del progreso tecnológico (que facilita bienes de consumo cada vez más atractivos a costes cada vez menores, siendo paradigmáticos los ejemplos proporcionados en el texto), y que era ese progreso el que resultaba proporcional al crecimiento económico global, por lo que éste, si bien de una forma algo menos directa, seguía resultando beneficioso al conjunto de la sociedad, y por ello seguía justificándose el paradigma de impuestos continuamente a la baja para los más ricos.

15 El valor agregado de una función multivariable se define como óptimo de Pareto, o Paretoóptimo, cuando no se puede aumentar el valor de una de las variables sin disminuir su valor para al menos alguna de las demás. En el caso de funciones de utilidad para múltiples agentes, se considera que han alcanzado su Pareto-óptimo cuando no se puede aumentar la utilidad de que disfruta algún agente sin disminuir aquella de la que disfruta otro (con independencia de las desigualdades que la distribución presente en el punto de "óptimo"). Aunque se le reconoce a Wilfredo Pareto el mérito de mover la ciencia económica del difuso sociologismo en que se encontraba en tiempos de Adam Smith a algo más basado en completas series estadísticas (y sus libros nos parezcan más "modernos", por la profusión de datos para soportar las premisas y el tratamiento formalmente matemático) no podemos olvidar que su "ley" de distribución de los recursos, que él creyó constante en todas las sociedades y todas las épocas, sirvió de inspiración a la política económica inicial de Benito Mussolini en Italia (del que Pareto fue en sus últimos años un entusiasta). La revisión rigurosa de los datos de que partió para la formulación de su ley en la segunda mitad del siglo XX hizo que fuese desechada y olvidada, pero la herramienta teórica del máximo que lleva su nombre sigue utilizándose en toda la teoría económica, y enseñándose en los primeros cursos de cualquier escuela. 
Incluso obviando el problema de la imposible neutralidad valorativa, una ciencia de la economía centrada en los comportamientos individuales se enfrenta a una dificultad que ninguna otra ciencia positiva encuentra: el carácter autoconsciente y libre de su objeto de estudio. Del hecho de que los hombres sean conscientes de sí mismos (y pueden por ello tomar decisiones basadas en las predicciones sobre su propio comportamiento de las que tengan noticia) se derivan dificultades predictivas bien conocidas (fenómeno sin paralelo en el resto del mundo natural de las profecías que se cumplen sólo en caso de haber sido enunciadas y difundidas, self fulfilling prophecies). El hecho de que los hombres sean libres ha hecho verter ríos de tinta sobre el verdadero alcance de nuestra voluntad y hasta qué punto es incondicionada, y sobre la posibilidad de compatibilizar esa libertad (aunque sólo sea considerada a priori como condición de posibilidad de nuestro comportamiento moral, supuesta por ello sin necesidad de pruebas empíricas a su favor, como postulaba Kant) con el conocimiento previo de nuestros patrones de comportamiento ${ }^{16}$. Independientemente de la posición que uno adopte frente al problema, podemos aceptar sin temor a la polémica que el reconocimiento de la dignidad humana nos exige tratar a las personas como si fuesen libres, responsables últimos de sus actos (salvo en casos de coacción evidente). Haya o no una cadena de causación irrompible detrás de cada movimiento que hacemos, para hacer el mundo humano inteligible debemos actuar como si esos movimientos no hubiesen podido en ningún caso ser predichos con certeza. Tomarnos en serio esa imposibilidad de predicción (y tenemos que tomárnosla en serio si reconocemos a nuestros semejantes como libres e iguales en dignidad a nosotros) es incompatible con tomarnos en serio el status científico de la economía ${ }^{17}$ o reconocer sus enunciados como verdades "de razón”.

\footnotetext{
${ }^{16}$ No es sorprendente que el primer tratamiento reconociblemente moderno de la posibilidad de compatibilizar la libertad humana con el conocimiento previo de nuestros actos -en este caso ese conocimiento previo es el que tiene Dios, gracias a su omnisciencia, de todo el devenir del universo- se lo debamos a Agustín de Hipona, el filósofo al que Arendt dedicó su tesis doctoral. En varios de los trabajos mencionados la autora asigna al romano la precedencia en la formulación del problema, que considera ausente en los autores tanto griegos como romanos anteriores. Véase por ejemplo el ensayo "What is freedom?” en Arendt (2006), pág. 144

17 Una última línea de defensa de la validez de los postulados a que llega la economía es la que podríamos llamar "analogía con la termodinámica" y viene a justificar sus conclusiones en la anulación de las irregularidades en el comportamiento de cada individuo al agregar el de muchos de ellos para poder predecir la evolución de un grupo suficientemente numeroso. Del mismo modo que el movimiento individual de una partícula de un gas es absolutamente estocástico y por ello (para todo sistema formado por más de unas pocas partículas) imposible de predecir, mientras que el comportamiento del gas en su conjunto es estrictamente determinista y predecible, se viene a postular que el comportamiento de cada sujeto aislado puede
} 
Nos hemos detenido más en la economía por tratarse de la ciencia social considerada metodológicamente más avanzada en su fundamentación y en el desarrollo de herramientas propias, pero no es mucho más sólida la situación del resto de disciplinas con aspiración de científicas nacidas en el tránsito del $S$ XIX al XX. Resulta interesante recuperar argumentos paralelos aplicados a la psicología de dos figuras de capital importancia para Arendt: su maestro Edmund Husserl y su colega y amigo durante toda su vida Hans Jonas. Así, en La crisis de las ciencias europeas y la fenomenología trascendental Husserl decía:

Se ha vuelto suficientemente claro para nosotros que una psicología "exacta", análoga a la física (esto es, el paralelismo dualista de realidades, de métodos y de ciencias), es un absurdo. Por ello no puede haber una psicología descriptiva que sea el análogo de una ciencia natural descriptiva. De ninguna manera, ni siquiera en el esquema de descripción versus explicación puede una ciencia del alma tomar como modelo las ciencias naturales o buscar consejo metodológico de ellas. ${ }^{18}$

Mientras que en El principio de responsabilidad, publicado en 1979, Jonas mantiene:

El hecho de que no tengamos "todavía" un conocimiento del hombre, la sociedad y la historia comparable al alcanzado por las ciencias naturales tiene el simple motivo de que no son "cognoscibles" en el mismo sentido en que lo es la "naturaleza", y que lo que es cognoscible de manera similar en ellos es bastante periférico a su verdadero conocimiento. ${ }^{19}$

ser asumido como estocástico sin que ello impida que se pueda agregar con el de otros muchos individuos para dar lugar a reglas igualmente deterministas, al cancelarse entre sí los efectos opuestos de las diferentes voluntades. Esta defensa se compatibiliza mal con el hecho de que la macroeconomía presenta un registro de aciertos en sus predicciones notablemente más pobre que el de la micro (aunque tal pobreza pueda achacarse en parte a la mayor humildad en las predicciones de esta última). El problema de este argumento es que no hay forma fiable de realizar esa agregación de comportamientos individuales de forma coherente, por la necesidad de tener en cuenta el "efecto rebaño" por el que los individuos modifican su comportamiento para adaptarlo al del grupo formando bucles de realimentación imposibles de modelar adecuadamente.

18 Husserl (1970), Parte III "La clarificación del problema transcendental y la función relativa de la psicología”, sección B "El camino a la filosofía fenomenológica trascendental desde la psicología”, \$65, pág. 223.

19 Jonas (1984). P 164. 
Resumiendo, por su incapacidad para situarse en el terreno de neutralidad axiológica que se debe exigir a una ciencia y por la incompatibilidad de sus posibles predicciones con el hecho tozudo y reiteradamente demostrado de la libertad humana podemos concluir que la economía no trata, tomando prestada la definición de Aristóteles, de lo necesario. Las reglas para decidir qué produce la sociedad, cómo lo distribuye y cómo lo remunera son enteramente contingentes, y es una expresión enteramente digna de la libertad humana el dedicar su ingenio a determinarlas. De hecho, si volvemos nuestra mirada a aquellas sociedades que sirvieron de modelo más directo a Arendt para modelar su visión del uso adecuado del espacio público (la polis ateniense y la comuna de París) veremos que dedicaban una parte importante del tiempo en el mismo a discusiones de naturaleza económica, como los tributos a pagar, las exenciones posibles y las obras a que se dedicarían esos ingresos.

Con ello consideramos suficientemente substanciada nuestra propuesta de que una sociedad más humana no necesita menos debate económico en el espacio público, sino más (lo que requiere en primer lugar de ciudadanos con conocimiento suficiente sobre las consecuencias de las decisiones económicas que tomen). El progreso en la construcción de una sociedad más justa y digna, más conducente al desarrollo de las capacidades más elevadas de sus miembros, es incompatible con el relegamiento de la economía al ámbito del hogar y la familia ${ }^{20}$. Sólo si suficientes ciudadanos pueden contribuir a la formación de las decisiones colectivas sobre qué producir y cómo repartir el fruto de esa producción se tendrán en cuenta de forma adecuada los diferentes intereses y se atenderá equitativamente a los diferentes colectivos. Quien pretenda disponer de un conocimiento especial (una techné) que le permite tomar esas decisiones sin la participación de la mayoría esconde generalmente el hecho de que las decisiones que tomará serán conducentes a su mayor bienestar (a costa del de algún otro grupo) sin que ello tenga necesariamente que ser así. Hemos refinado con ello el primer presupuesto de la crítica de Arendt a la evolución del espacio público en las sociedades modernas (su colonización por consideraciones económicas,

${ }^{20}$ Lo que no quiere decir que ese relegamiento o su equivalente sustracción al ámbito público para ser confinada en camarillas de expertos por su supuesta complejidad no se hayan intentado. La separación de economía y sociedad, o el intento de establecimiento de un mercado de reglas no sujetas a discusión colectiva es precisamente lo que se denuncia en Polanyi (2001). 
entendiendo la economía como una ciencia positiva). A la revisión del segundo presupuesto volvemos ahora nuestra atención.

\section{La soportable levedad de la política}

Sabemos de la profunda desconfianza de Arendt por las todavía jóvenes "ciencias sociales", en sus propias palabras:

Las comparativamente nuevas ciencias sociales, que tan rápidamente se han convertido para la historia en lo que la tecnología ha sido para la física, pueden usar la experimentación en un sentido mucho más crudo y menos fiable que las ciencias naturales, pero el método es el mismo [...] Si su vocabulario es repulsivo y su esperanza de cerrar la supuesta brecha entre nuestro dominio científico de la naturaleza y nuestra deplorada impotencia para "gestionar" los asuntos humanos a través de una ciencia de la ingeniería de las relaciones humanas suena aterradora es sólo porque han decidido tratar al ser humano como una criatura enteramente natural, cuyos procesos vitales pueden ser manipulados exactamente igual que cualquier otro proceso. ${ }^{21}$

Más adelante en el mismo ensayo se lamentará de que las técnicas basadas en esas ciencias, cuyo campo de experimentación identifica en los países totalitarios, terminarán por hacer al mundo de las relaciones y los asuntos humanos lo que la tecnología basada en la física y la química ha sido ya capaz de hacer para el mundo de los artefactos ${ }^{22}$. Semejante desconfianza se origina en una visión de la política orientada a un fin muy específico (e incompatible con el recurso a ciencias que traten sólo de lo necesario, o cuyos enunciados puedan considerarse "de razón" y no sujetos por ello a discusión legítima): el mantenimiento de un espacio público en que los hombres puedan "actuar” espontáneamente, mostrarse

${ }^{21}$ En "The concept of history", 20 ensayo contenido en Arendt (2006), pág. 59.

${ }^{22}$ Op. Cit. pág. 89, si bien la visión del desarrollo y peligros de la economía, sociología y psicología que se practicaban en los países comunistas que se tenía desde dentro de los mismos era bastante menos optimista. Según nos narra Leszek Kolakowski en su imprescindible Main Currents of Marxist (Vol. 3, The Breakdown) en los tiempos en que Arendt expresaba esas inquietudes tanto en Rusia como en Alemania del Este, Polonia y el resto de países del bloque soviético las ciencias sociales eran una sombra de lo que habían sido, bajo el férreo control de la censura estatal y limitadas a una hermenéutica tan dogmática como estéril de los textos fundacionales aceptados como indiscutiblemente válidos. 
unos a otros en su individualidad y persuadirse (excluyendo por ello el recurso a la violencia o la apelación a una soberanía en que unos deban necesariamente obedecer las órdenes de otros) para realizar acciones conjuntas dignas de ser recordadas. Acciones que sólo cobran sentido en ese mundo artificial, distinto del natural (y separado del mismo por fronteras bien definidas), que los hombres construyen a su alrededor.

En su obra posterior ${ }^{23}$ Arendt nos dirá que la reflexión política empieza en Occidente a partir de un hecho muy preciso: la condena a muerte de Sócrates, que lleva tanto a Platón como a Aristóteles a plantear la necesidad de un espacio privado, exento de las obligaciones de la vida pública, en que los pensadores puedan perseguir la búsqueda de la verdad de forma tranquila. Desde entonces la práctica política se considera no solo opuesta (tanto el archein/ agere como el prattein/gerere que constituyen el núcleo y está en el origen filológico de esa práctica para Arendt pasan a identificarse como diferentes e incompatibles con el scholél otium imprescindible para poder pensar correctamente), sino inferior a la tranquilidad de espíritu exigida por la actividad filosófica (así, Aristóteles expone la superioridad del bios theoretitikós o vida dedicada a la contemplación sobre el bios politikós o vida dedicada al grupo y sus asuntos). De forma similar a como la actividad política presupone el que los participantes en la misma tengan sus necesidades vitales resueltas (tanto en el ámbito de la labor, para lo que podían contar con mujeres y esclavos, como en el del trabajo, reflejado en el rechazo a los banausoi o artesanos para participar en las decisiones de la polis) la actividad puramente intelectual requerirá que el grupo esté mínimamente ordenado, al menos para no interferir con sus imposiciones y su violencia en la vida contemplativa del filósofo.

Ahora bien, aún más interesante que la consideración arendtiana sobre el inicio de la reflexión filosófica sobre la política resulta su opinión sobre el momento en que ésta llega a su fin, con la obra de Karl Marx, tras el que si seguimos a la autora no queda ya más que pensar filosóficamente sobre la misma. El motivo de dicho "cierre" de la tradición es que poco después de la revolución industrial el trabajo adquiere un carácter "social": pasa a realizarse en un espacio separado del ámbito privado de la familia y el hogar que sin embargo se diferencia del espacio público, ya que en él los participantes no pueden presentarse como iguales y

23 Principalmente en el artículo "Introducción a la política”, que podemos encontrar tanto en Arendt (2003) como en Arendt (2008), aunque también en "Tradition and the modern age" y "What is authority?" en Arendt (2006). 
se someten a un proceso predefinido que les impide manifestar su personalidad o actuar espontáneamente. Por otro lado, toma elementos hasta entonces exclusivos de la labor, como el hecho de que su producto pase a ser perecedero y rápidamente desechable. La teoría marxista no hace más que constatar esta situación novel, y desvelar cómo lleva asociada la necesaria pérdida de valor de las actividades humanas (ya que el valor tanto de las cosas como de las acciones se otorga necesariamente por el conjunto social, no es algo que el individuo pueda producir aisladamente). Una de las consecuencias de esa pérdida de valor, de la trasposición de la dinámica de la labor a todas las áreas de la vida, es que la sociedad pasa a "consumir" incluso aquellos elementos originalmente destinados a perdurar (desde el mismo producto del trabajo hasta las producciones culturales, que al adaptarse a las masas necesariamente se degradan). Sin embargo y paradójicamente, aunque ese trabajo socializado limita su acceso al espacio público, el avance tecnológico que se deriva de él tiene como consecuencia la automatización de los procesos productivos necesarios para mantener el mundo artificial del que necesitamos rodearnos (mundo compuesto precisamente por los objetos fruto del trabajo), por lo que la cantidad total de trabajo necesario no para de disminuir, creando el problema inédito de qué harán las masas desocupadas (para las que Arendt comparte el diagnóstico común a Platón y Aristóteles de que difícilmente se sentirán satisfechas dedicando su vida a la persecución de la verdad o la belleza).

Afortunadamente, el cierre de la tradición que Arendt identifica en el pensamiento de Marx no le lleva a desistir en su propia reflexión sobre la política, una reflexión fructífera y relevante, en la que insiste en la necesidad de asegurar ese espacio común en que los hombres se presentan como iguales a la hora de escuchar y ser escuchados, en el que coordinan sin coacción sus acciones y que les permite conseguir grandes cosas, dignas de ser recordadas sin necesidad de que un gran poeta como Homero las tenga que embellecer ${ }^{24}$. Podemos imaginar que en nuestros propios días Arendt identificaría como apropiadas para el espacio público (y sujeto legítimo por ello de la actuación política) la discusión sobre quiénes forman en primer lugar el cuerpo social, y tienen por ello el derecho de ser escuchados, mientras que miraría con sospecha la dedicación casi exclusiva

${ }^{24}$ El discurso, recogido por Tucidides, en que Pericles expone la gloria de Atenas basándose en este argumento es especialmente querido para Arendt, que lo utiliza en numerosos ensayos, como "The concept of history", pág. 72; "The Crisis in Culture", pág. 213; ambos en Arendt (2006), en "Introducción a la política”, pág. 158-9 en Arendt (2008), y por supuesto en el capítulo V dedicado a la acción en Arendt (1998). 
de los parlamentos nacionales a discutir sobre el presupuesto anual y el mejor destino de las subvenciones.

En tiempos de graves desplazamientos de los desheredados de la tierra (que reclaman no sólo ser atendidos en su necesidad urgentísima, sino reconocidos de forma que pueden tener un horizonte de esperanza más allá de dicha atención) y de tensiones secesionistas en alguno de los territorios históricos de nuestro país, es indudable que nos beneficiaríamos de una discusión más política en el sentido Arendtiano, de dedicar más tiempo en común, pasado en el espacio público accesible a todos, en discutir persuasivamente de éstos asuntos. Sin embargo el circunscribir el discurso político exclusivamente a cuestiones de esa importancia puede debilitarlo de otra manera, vaciándolo de contenidos relevantes y relegándolo a la mera discusión sobre gustos estéticos, pues la polis griega no es el único ejemplo de sociedad en la que los individuos se encontraban para hacer grandes hechos y decir grandes palabras, y algunas de las alternativas podrían no parecernos tan atractivas ${ }^{25}$.

\section{Conclusiones: Globalización y economía del conocimiento}

Las circunstancias históricas en que Arendt escribió La Condición Humana contenían ya la semilla de lo que hoy conocemos como posmodernidad (aunque el término parece haber caído en desuso y hoy se oiga más hablar de "globalización"). La recién terminada guerra mundial constataba la quiebra del modelo de racionalidad definido por la Ilustración, quiebra de la que todavía estamos intentando recomponer los pedazos. Sin embargo, al contrario de lo ocurrido con los discursos para intentar dar razón de la situación del hombre en el mundo, que la guerra parecía haber vuelto banales (porque el propio discurso se consideraba deslegitimado, y su elaboración una actividad poco de fiar), la ciencia y la técnica a las que la guerra dio un empujón muy considerable no han dejado de avanzar, cambiando modos y costumbres en su desarrollo. Parte de

\footnotetext{
25 Nos referimos a la sociedad del Japón feudal descrito por la dama Murasaki Shikibu en su Historia de Genji, en que los innumerables nobles que protagonizan la historia, teniendo sus necesidades vitales tan cubiertas como los ciudadanos libres atenienses, discurren sus vidas discurseando sin parar sobre... nada en absoluto, al considerar los asuntos económicos indignos de su tiempo, y estar éstos suficientemente atendidos por una administración eficiente. Tan eficiente como para ejercer sobre ellos un poder omnímodo e incontestable que nos hace cuestionarnos hasta dónde llegaba realmente su percibida libertad.
} 
estos avances (automatización de la producción industrial) eran ya visibles para Arendt, que se preguntaba qué pasaría en un mundo en que el trabajo estaba desplazando a la acción mientras que a su vez éste iba siendo inexorablemente desplazado por la técnica y viéndose obligado a tomar características de la labor. En parte veía cómo el fruto de ese trabajo, que originalmente se diferenciaba por su "aspiración de eternidad", por estar diseñado para durar, se haría más y más fungible, más y más orientado a ser consumido y reemplazado. Tenía razón en que el coste para el planeta de una sociedad de consumo que mantiene su ritmo de crecimiento a base de ciclos de obsolescencia cada vez más cortos terminaría por hacerse insostenible (y las consecuencias de que deje de haber trabajo para los seres humanos que han dejado de saber vivir actuando porque sólo saben trabajar son nefastas). Ya Marx había advertido de cómo la dinámica del capitalismo exigía a las empresas hacer crecer su producción de forma continua, lo que, a falta de incrementar el poder adquisitivo de las masas trabajadoras (cuyos salarios se mantenían al nivel necesario para la mera subsistencia por la existencia de un "ejército de reserva" de desempleados), necesitaba continuamente de nuevos mercados. Por eso en su visión el capitalismo tenía que conducir necesariamente al colonialismo y finalmente sucumbir ante crisis cada vez más violentas forzadas por la persecución de una tasa de retorno inevitablemente decreciente. Pero en un mundo enteramente colonizado ya no hay cómo dar salida a la creciente producción incorporando a nuevos pueblos a la espiral de consumo, el mercado alcanza una dimensión fija, e incluso el crecimiento de la población, que durante décadas había seguido nutriendo abundantemente de consumidores a las multinacionales, parece estar finalmente remitiendo ${ }^{26}$. ¿ Presagia esta remisión que la única alternativa para la continuación de nuestro sistema de producción (eliminada la posibilidad de crecimiento por conquista de nuevos mercados o por aumento "natural" de la población) es efectivamente reponer cada vez más rápido los bienes por ello llamados "de consumo", sea cual sea el coste para el medio ambiente? La cuestión merece considerar cómo ha cambiado la sociedad entre nuestro tiempo y el de Arendt.

De una economía industrial hemos pasado primero a una de servicios y luego a una basada en el intercambio de información. Dos consecuencias positivas de

${ }^{26}$ El colapso mundial de las tasas de fertilidad, que alcanza a todas las sociedades en cuanto llegan a un cierto nivel de desarrollo económico (y que falta ya por materializarse únicamente en las naciones más pobres de África, siendo ya constatable tanto en Asia como en Latinoamérica), apunta a una estabilización de la población total del planeta a mediados de este siglo cuando se alcancen los nueve mil millones de habitantes, véase Longman (2004). 
ese cambio son que la actividad remunerada de un "trabajador del conocimiento" es difícilmente replicable por una autómata (lo que atenúa la zozobra de tener que determinar a qué se pueden dedicar las masas de trabajadores a las que la automatización dejaría sin trabajo) y que no requiere ya del consumo de recursos finitos (materias primas), por lo que su impacto en el medio ambiente es mucho menor $^{27}$. No podemos esperar que Arendt identificase estas posibilidades en su tiempo, y podemos considerarlas un ejemplo de ese concepto de "nuevos comienzos" que ella consideraba inherente a la condición humana, simbolizado en la posibilidad de "traer algo nuevo al mundo" pero también en la imposibilidad de predecir cuáles irían a ser las consecuencias de nuestras acciones ${ }^{28}$.

El garantizar que no se perdía esa posibilidad de nuevos comienzos era para ella la finalidad más alta de la acción política, y por eso definió ésta de forma que excluyese de su ámbito toda disciplina que nos hiciese creer que las consecuencias de nuestros actos estuvieran determinadas, fuesen necesarias. Sin embargo, como esperamos haber demostrado, esta exclusión está basada en consideraciones epistemológicas inciertas (sobre el tipo de conocimiento que materias como la economía y la psicología pueden proporcionar) y, de aplicarse, corre el riesgo de vaciar el discurso político (y el ámbito en que éste se desarrolla, el espacio público) de elementos que necesita ponderar precisamente para no volverse él mismo

27 En el capítulo 5, el menos inspirado de El principio de responsabilidad, Hans Jonas argumentaba la superioridad moral de un régimen autocrático como el comunismo porque sería el único capaz de imponer los grandes sacrificios requeridos para "alinear el nivel de vida por abajo" de forma que los habitantes del primer mundo viviesen tan mal como los del tercero (un ejemplo de tarea hercúlea de ese gobierno autoritario sería cerrar las fábricas del primer mundo y abrirlas en el tercero), única forma de vida compatible (y aún eso con dudas) con la supervivencia de la especie. Criticaba especialmente la visión de Ernst Bloch (el título de la obra de Jonas, según él mismo, es una refutación del Das prinzip Hoffnung de Bloch) de que la tecnología nos permitiría alimentar a una humanidad muchas veces mayor que la actual que viviría en una utopía libre de trabajo. Los hechos parecen estar dando la razón más a Bloch que a Jonas, ya que la producción industrial de América y Europa se trasladó a China, Vietnam, Corea, India, etc. sin que ningún gobierno autoritario lo tuviera que imponer, la población se está estabilizando de forma igualmente espontánea, y los avances en agricultura y ganadería (la segunda "revolución verde" de los 70) permiten que las hambrunas que aún se producen y se puedan producir en el futuro sean antes causadas por problemas de distribución (normalmente debidos a líderes autocráticos corruptos) que de insuficiencia de la producción.

${ }^{28}$ Como reflejamos en la nota 15 esa concepción de la libertad como algo incompatible con la predicción de nuestras acciones es de origen claramente agustiniana, con claros paralelismos con lo que Alvin Plantinga, otro filósofo fuertemente influenciado por la idea agustiniana de la libertad denominaría "libertarianismo" (libre albedrío incompatible con ningún determinismo). 
una discusión superflua, mientras que las decisiones que realmente importan, que realmente van a determinar si seguimos disfrutando de la libertad de comenzar cosas nuevas e impredecibles, se toman en otros foros y sin nosotros.

\section{Bibliografía}

Arendt, Hannah (1976). The Origins of Totalitarianism. Orlando: Harcourt Inc, ed. Original de 1951

(1998). The Human Condition. Chicago: The University of Chicago Press, ed. Original de 1958

(2006). Between Past and Future. Nueva York: Penguin, con introducción de Jerome Kohn, ed. Original de 1961

(2006b). On Revolution. Nueva York: Penguin, ed. Original de 1963

(1981). The Life of the Mind. Orlando: Harcourt Inc, editado de forma póstuma por Mary McCarthy.

(2003). Was ist Politik?. Múnich: Piper Verlag, editado por Ursula Ludz de forma póstuma originalmente en 1993

(2008). La promesa de la política. Barcelona: Paidós, editado y con introducción de Jerome Kohn, traducido por Eduardo Cañas y Fina Birulés

Birmingham, Peg (2006). Hannah Arendt and Human Rights. Bloomington: Indiana University Press

Birulés, Fina (2009). Una herencia sin testamento: Hannah Arendt, Barcelona: Herder.

Canovan, Margaret (1994). Hannah Arendt. A reinterpretation of her political thought. Cambridge, Cambridge University Press

Dilthey, Wilhelm (1988). Introduction to the Human Sciences. An Attempt to Lay a Foundation for the Study of Society and History. Detroit: Wayne State University Press, traducción de Ramón J. Betanzos de la edición original de 1923

Husserl, Edmund (1970). The Crisis of European Sciences and Trascendental Phenomenology. An Introduction to Phenomenological Philosophy. Evanston: Northwestern University Press, traducción de David Carr de la edición original de 1954

Jonas, Hans (1984). The Imperative of Responsibility. In Search of an Ethics for the Technological Age. Chicago: The University of Chicago Press, traducción del propio Jonas con la colaboración de David Herr de la edición original de 1979 
Kunn, Thomas S. (1996). The Structure of Scientific Revolutions 3rd Edition. Chicago: The University of Chicago Press, ed. Original de 1962

Longman, Philip (2004). The Empty Craddle. How Falling Birthrates Threaten World Prosperity [and what to do about it]. New York: Basic Books.

Platón (2012). La república. Madrid: Alianza Editorial

Polanyi, Karl (2001). The Great Transformation. The Political and Economic Origins of Our Time. Boston: Beacon Press, ed. Original de 1944

Young-Bruehl, Elizabeth (2004). Hannah Arendt: for Love of the World. New Haven: Yale University Press, ed. Original de 1982

Recibido: 14/07/2015

Aceptado: 14/10/2015

Este trabajo se encuentra bajo una licencia de Creative Commons ReconocimientoNoComercial-SinObraDerivada 4.0 
